\title{
REPORTE DE CASOS
}

\section{Infecciones por Staphylococcus aureus meticilino resistentes adquíridas en la comunidad}

\section{Community-acquired methycillin-resistant Staphylococcus aureus infections}

\section{María Claudia Ibarra Orrego ${ }^{1}$, Néstor Arrúa Torreani², Carlos Kunzle Durañona ${ }^{3}$, Anacleto Acuña ${ }^{4}$}

\begin{abstract}
${ }^{1}$ Postgrado en Medicina Interna. Facultad de Ciencias Médicas. Universidad Nacional de Asunción (AsunciónParaguay)

${ }^{2}$ Tutor Docente. Universidad Nacional de Asunción (Asunción- Paraguay)

${ }^{3}$ Infectólogo Docente del Servicio de Clínica Médica del Hospital Militar Central de las Fuerzas Armadas (AsunciónParaguay)

${ }^{4}$ Jefe de sala y Tutor Docente del Servicio de Clínica Médica del Hospital Militar Central de las Fuerzas Armadas (Asunción-Paraguay)
\end{abstract}

\section{RESUMEN}

Se presentan 8 pacientes adultos internados en el Servicio de Clínica Médica del Hospital Militar Central (Asunción, Paraguay) entre enero/2014 y julio /2016 con infecciones pulmonares, de piel y partes blandas producidas por $S$. aureus resistentes a la meticilina adquiridas en la comunidad (SARMAC). El 87,5\% presentó bacteriemia, de los cuales la mitad evolucionó desfavorablemente, requiriendo internación en la Unidad de Terapia Intensiva.

Palabras claves: Staphylococcus aureus, infecciones estafilocócicas, resistencia a la meticilina adquiridas en la comunidad

\begin{abstract}
We present 8 adult patients admitted to the Medical Clinic of the Central Military Hospital (Asunción, Paraguay) between January, 2014 and July, 2016 with lung, skin and soft tissues infections caused by community-acquired methycillin-resistant Staphylococcus aureus (CA-MRSA). Bacteremia was present in $87,5 \%$, half of them evolved unfavorably requiring admission into the Intensive Care Unit.
\end{abstract}

Keywords: Staphylococcus aureus, staphylococcal infections, methycillin resistance, communityacquired

\footnotetext{
Autor correspondiente:

Dra. María Claudia Ibarra Orrego.

Dirección: Ruta 1 km 62. Paraguarí- Paraguay

Teléfono: +595.986250169

Correo electrónico: maclaudiaiba88@gmail.com
}

Artículo recibido: 29 diciembre 2016

Artículo aceptado: 6 febrero 2017 


\section{INTRODUCCIÓN}

Los estafilococos son microorganismos aerobios gram positivos. El más patogénico de ellos es el Staphylococcus aureus, que típicamente causa infecciones de la piel y a veces neumonía, endocarditis y osteomielitis. En general se lo asocia con la formación de abscesos. Algunas cepas elaboran toxinas que causan gastroenteritis, síndrome de la piel escaldada y síndrome de shock tóxico. El diagnóstico se realiza con tinciones de Gram y cultivos.

Su protagonismo ha ido creciendo en los últimos años por la aparición de una nueva cepa, $S$. aureus meticilino resistente, independientemente de la forma de adquisición. Hasta hace poco era considerado patógeno principalmente nosocomial, hoy cobra real importancia como patógeno comunitario $^{(1,2)}$.

Hacia 1940 se inició el uso clínico de la penicilina, siendo el 95\% de las cepas sensibles a la misma. A comienzos de la década del 50 esta sensibilidad se había reducido en $50 \%$, a causa de la síntesis de betalactamasas como mecanismo productor de resistencia antibiótica. Con el fin de contrarrestar el efecto de estas enzimas, hacia 1960 aparece la meticilina. A tan sólo un año de su utilización se comunican las primeras cepas multirresistentes asociadas a determinados factores de riesgo: internaciones en cuidados intensivos, pacientes en diálisis, cirugías, procedimientos invasivos, etcétera.

En la década de 1990 se comunicó la aparición de cepa de S. aureus sin los factores de riesgo clásicos para la adquisición de SAMR. Estas cepas se denominaron $S$. aureus meticilino resistente adquirido en la comunidad (SAMR-AC). Tienen un patrón de susceptibilidad antimicrobiana diferente al de las cepas de SAMR hospitalarias, siendo susceptibles al trimetroprimsulfametoxazol, gentamicina, clindamicina y vancomicina ${ }^{(2,3)}$.

EI SAMR-AC es capaz de producir una penicillin binding proteins (PBP) distinta llamada PBP2a, con baja afinidad por los betalactámicos que determinan la resistencia a la meticilina ${ }^{(4,5)}$. Las codificaciones para esta PBP2a residen en un gen cromosómico, llamado mec A.

Este gen se encuentra en un elemento genético móvil llamado casette cromosómico estafilocócico mec tipo IV. Este nuevo casette cromosómico SCC mec tipo IV encontrado en el SAMR-AC es distinto de los tres ya descriptos anteriormente, para las cepas de SAMR hospitalarias. A diferencia de los anteriores, no posee otros genes de resistencia que el gen $\operatorname{mec} A^{(5,6)}$.

Otro aspecto que diferencia al SAMR-AC es su comportamiento frente a los antibióticos macrólidos. Los SAMR-AC con resistencia a la eritromicina en general son sensibles a la clindamicina. Pueden presentar porcentajes variables de resistencia inducible a la clindamicina, que es diferente según distintos países y regiones. También se les atribuye mayor poder de virulencia e invasividad por la presencia de una toxina "killing leucocyte", codificada por el factor Panton Valentine Leukocidine ${ }^{(7)}$.

El tratamiento suele incluir betalactámicos resistentes a las penicilinasas, pero como es frecuente la resistencia a antibióticos pueden ser necesarios vancomicina u otros fármacos más modernos. Algunas cepas son parcial o totalmente resistentes a todos los antibióticos excepto los más nuevos, como la linezolida, la asociación quinupristina/dalfopristina, la daptomicina, la telavancina, la dalbavancina y la tigeciclina.

En el país, entre en el 20 y $30 \%$ de las infecciones causadas esta cepa requieren el uso de vancomicina. Aun no se ha descrito resistencia de ninguna cepa de esta bacteria ${ }^{(8)}$. 
Doi:10.18004/rvspmi/2312-3893/2017.04(01)100-104

Es causante desde infecciones leves de la piel como son el impétigo y los furúnculos hasta cuadros muy graves como la neumonía, infección de huesos y articulaciones, meningitis, septicemia, endocarditis, cuadros que conllevan a alta mortalidad.

Ante la comunicación del primer aislamiento de $S$. aureus resistente a la vancomicina en América Latina, ocurrida años atrás en Brasil, la OPS/OMS recomienda a los Estados Miembros continuar con el establecimiento y el mantenimiento de sus capacidades para la rápida detección y notificación de este mecanismo de resistencia a los antibióticos, para el establecimiento de medidas de prevención y control de las infecciones asociadas a la atención de salud ${ }^{(8)}$.

\section{REPORTE DE CASOS}

Se estudiaron 8 casos correspondientes a la totalidad de pacientes con cultivos positivos a SAMRAC durante un período de 30 meses. La distribución por edades fue entre 18 años a $\geq 60$ años (tala 1). Todos fueron de sexo masculino.

Los casos correspondientes entre 18 -23 años de edad, se presentaron sin comorbilidades, uno de ellos con cuadro respiratorio, fiebre y una forunculosis abscedada en brazo izquierdo. Fue internado con el diagnóstico de neumonía adquirida en la comunidad bilateral. El otro paciente se presentó con antecedentes de traumatismo de tórax cerrado sin cuadro respiratorio, el diagnóstico fue realizado radiológicamente.

Entre los pacientes $>30$ años y $<60$ años, uno tenía diabetes mellitus y tuvo como puerta de entrada un forúnculo nasal, evolucionando a una neumonía con empiema pleural. El otro caso tuvo como puerta de entrada múltiples inyecciones intramusculares evolucionando a un absceso glúteo derecho. El tercer paciente de este grupo etario se trató de un diabético con una infección urinaria a $S$. aureus cursando con bacteriemia.

El grupo de >60 años eran pacientes con comorbilidades como hipertensión arterial y diabetes mellitus con sus complicaciones macro y microangiopáticas. Un caso presentó bacteriemia a punto de partida urinario, otro a punto de partida de un traumatismo abierto en miembro inferior y el tercero tuvo una erisipela sin puerta de entrada evidenciable. (Tabla 1).

Tabla 1

Distribución según edad ( $\mathrm{n}$ 8)

\begin{tabular}{lcc}
\hline Edad (años) & No & \% \\
\hline $18-23$ & 2 & 25 \\
$36-41$ & 1 & 12,5 \\
$42-47$ & 1 & 12,5 \\
$48-53$ & & \\
$54-59$ & 1 & 12,5 \\
$=60$ & 3 & 37,5 \\
\hline
\end{tabular}

Todos estos casos adquirieron la infección en la comunidad. La mayoría procedía de zonas rurales (tabla 2). 
Tabla 2

Forma de adquisición de la infección y procedencia de los pacientes

\begin{tabular}{ccc}
\hline Adquisición & & Procedencia \\
\hline Comunidad & Urbana & Rural \\
8 & 5 & 3 \\
$100 \%$ & $62,5 \%$ & $37,5 \%$ \\
\hline
\end{tabular}

De la muestra, 6 casos se aislaron SARM (75\%) y en 2 casos se aislaron SASM (25\%). El 25\% eran pacientes con comorbilidades, sin esto tener relación con la gravedad de los casos. Se consideraron episodios graves la presencia de hipotensión arterial, insuficiencia respiratoria $\left(\mathrm{PO}_{2}<60 \%\right)$ neumotórax, derrame pleural. Del total de la muestra, $87 \%$ presentó hemocultivos positivos.

Independientemente de tener cultivos positivos de otras secreciones para el mismo germen, solo 1 caso resultó con hemocultivo positivo, pero en el resto de las secreciones se aisló al SARM. En 62,5\% se pudo identificar la piel como puerta de entrada, en $25 \%$ se trató de las vías urinarias y solo en $12,5 \%$ no se identificó la puerta de entrada. En $50 \%$ evolucionaron con complicaciones (empiemas y casos donde hubo compromiso hemodinámico).

Solo $37,5 \%$ recibió tratamiento empírico con vancomicina, resultando el tratamiento correcto al recibir los resultados del antibiograma, que demostraron resistencia a la meticilina. El $62,5 \%$ fue tratado inicialmente en forma empírica con antibióticos betalactámicos, pero al recibirse el antibiograma de los cultivos se demostró que el $S$. aureus era resistente a dichos antibióticos por lo que hubo necesidad de rotar a vancomicina o a trimetroprim-sulfametoxazol. El $75 \%$ requirió algún tipo procedimiento quirúrgico (instalación de tubo pleural, drenaje de abscesos, etc.).

\section{DISCUSIÓN}

En el Servicio de Clínica Médica se obtuvieron ocho cultivos a SAMR-AC en un lapso de 30 meses. En los últimos años se ha producido la aparición e incremento de infecciones causadas por $S$. aureus resistentes a meticilina provenientes de la comunidad ${ }^{(9-11)}$. Este hecho puede atribuirse al aumento de las infecciones de piel en épocas de calor.

Según los datos del país, entre 20 y 30\% de las infecciones causadas por esta cepa requieren el uso de vancomicina. Aun no se ha descrito resistencia de $S$. aureus, datos que se comprobaron con este estudio.

En los casos descritos hubo cuatro pacientes con infecciones invasivas por SAMR-AC. Tuvimos dos casos de neumonía con empiema por SAMR-AC, aunque el $S$. aureus rara vez ha sido reportado como el agente causal, sin embargo, la mayoría son sensibles a meticilina ${ }^{(12)}$, situación que en este caso no se dio, pues ambos resultaron ser SARM.

No se realizó búsqueda de estado portador nasal ni tampoco el tratamiento de descolonización, pues en la literatura internacional no hay consenso que justifique la utilización rutinaria del tratamiento de descolonización nasal en estados de portador a SAMR-AC en la población general ${ }^{(13-16)}$.

En muchos casos el tratamiento antibiótico tópico y/o drenaje quirúrgico es suficiente en la mayoría de las infecciones a SAMR-AC, siempre teniendo en cuenta el patrón de resistencia antibiótica de este germen, a la hora de comenzar antibioticoterapia en forma empírica. 
Es importante hacer hincapié en la solicitud del estudio bacteriológico de las lesiones, así como en el seguimiento del paciente, para realizar una evaluación correcta del tratamiento indicado. Esto evitaría las múltiples consultas y la heterogeneidad de tratamientos realizados en forma empírica ${ }^{(7,13,14)}$.

\section{REFERENCIAS BIBLIOGRÁFICAS}

1. Stevens MP, Edmond MB. Staphylococcus aureus. In: Wenzel R, Bearman G, Brewer T, Butzler JP, editors. A guide to infection control in the hospital. 4th ed. Boston: ISID; 2008. p. 241-6. Disponible en: http://beta.isid.org/downloads/GuideInfControlHospital4thEd.pdf

2. Naimi TS, LeDell KH, Boxrud DJ, Groom AV, Steward CD, Johnson SK, et al. Epidemiology and clonality of community-acquired methicillin-resistant Staphylococcus aureus in Minnesota, 19961998. Clin Infect Dis. 2001; 33(7):990-6.

3. Picazo JJ, Betriu C, Rodríguez-Avial I, Azahares E, Ali Sánchez B. Surveillance for antimicrobial resistance: VIRA study. Enferm Infecc Microbiol Clin. 2002; 20(10):503-10.

4. Savio E, Medina J, Hernández O. Emergencia de un nuevo Staphylococcus aureus meticilinoresistente comunitario (SAMR-com) con un perfil más angosto de resistencia. Montevideo: OPS; 2004.

5. Zetola N, Francis JS, Nuermberger EL, Bishai WR. Community-acquired meticillin-resistant Staphylococcus aureus: an emerging threat. Lancet Infect Dis. 2005; 5(5):275-86.

6. Katayama $Y$, Ito T, Hiramatsu K. A new class of genetic element, staphylococcus cassette chromosome mec, encodes methicillin resistance in Staphylococcus aureus. Antimicrob Agents Chemother. 2000; 44(6):1549-55.

7. Marcinak JF, Frank AL. Treatment of community-acquired methicillin-resistant Staphylococcus aureus in children. Curr Opin Infect Dis. 2003; 16(3):265-9.

8. Arbo A. Infecciones adquiridas en la comunidad causadas por Staphylococcus aureus resistente a la oxacilina: representa una problemática en el Paraguay?. Rev Inst Med Trop. 2008; 3(1):6-8.

9. Kaplan SL. Treatment of community-associated methicillin-resistant Staphylococcus aureus infections. Pediatr Infect Dis J. 2005; 24(5):457-8.

10. Gonzalez BE, Martinez-Aguilar G, Hulten KG, Hammerman WA, Coss-Bu J, Avalos-Mishaan A, Mason EO Jr, Kaplan SL. Severe Staphylococcal sepsis in adolescents in the era of communityacquired methicillin-resistant Staphylococcus aureus. Pediatrics. 2005; 115(3):642-8.

11. Buckingham SC, McDougal LK, Cathey LD, Comeaux K, Craig AS, Fridkin SK, Tenover FC. Emergence of community-associated methicillin-resistant Staphylococcus aureus at a Memphis, Tennessee Children's Hospital. Pediatr Infect Dis J. 2004; 23(7):619-24.

12. Mizell KN, Patterson KV, Carter JE. Empyema necessitatis due to methicillin-resistant Staphylococcus aureus: case report and review of the literature. J Clin Microbiol. 2008; 46(10):3534-6.

13. Prego J, Galiana A, Pujadas M, Almada K, Boulay M, Carugati MJ, et al. Infecciones de piel y partes blandas en pacientes ambulatorios. Arch Chil Pediatr. 2006; 77(2):196-7.

14. Eady EA, Cove JH. Staphylococcal resistance revisited: community-acquired methicillin resistant Staphylococcus aureus--an emerging problem for the management of skin and soft tissue infections. Curr Opin Infect Dis. 2003; 16(2):103-24.

15. Chen SF. Staphylococcus aureus decolonization. Pediatr Infect Dis J. 2005; 24(1):79-80.

16. Mendoza C, Barrientos C, Panizza V, Concha B, Romero P, Barahona C, et al. Prevención de la infección intrahospitalaria por Staphylococcus aureus resistente a meticilina mediante el manejo de portadores. Rev Chil Infectol. 2000; 17(2): 129-34. 